\title{
Reporting Systematic Review in Accordance With the PRISMA Statement Guidelines: An Emphasis on Methodological Quality
}

\author{
Mehrdad Amir-Behghadami, MSc (10; Ali Janati, PhD
}

Key Words: methodological quality, PRISMA statement guidelines, systematic review

$\mathrm{W}$ e read with great interest the publication titled, "Use of Simulated Patients in Disaster Medicine Training: A Systematic Review," by Pier Luigi Ingrassia et al., ${ }^{1}$ in the journal, Disaster Medicine and Public Health Preparedness. Although the authors have stated that they reported according to the Preferred Reporting Items for Systematic Reviews and Meta-Analyses (PRISMA) guidelines, some items have not been well reported. Therefore, the aim of this letter is to present methodological issues about the search strategy and quality assessment of included studies.

First, the number of databases searched for literature has been limited to PubMed, the search strategy seems simple, and the only language of interest is English. However, other databases could be searched. This may increase the likelihood of a search bias, that is, missing some studies, language bias, and publication bias. Searching a single database can reduce sensitivity to as low as $66 \%$. Generally, a systematic review must search major medical databases, including PubMed, Scopus, Web of Science, EMBASE, and Cochrane Library. ${ }^{2,3}$ Also, according to the PRISMA statement, it is suggested that the search strategy be devised at least for PubMed and replicated for the other electronic databases. ${ }^{4}$

Second, despite not making any qualitative evaluations possible in this study, we observed that none of the studies included in this review have been qualitatively evaluated. Analyzing and interpreting preliminary studies in a systematic review require qualitative assessment and bias sensitivity assessment because poor quality studies can affect the quality of the results and distort the results of the studies. ${ }^{5,6}$ The included studies should be evaluated with tools that are appropriate to the type of study. Determining the type of study in the inclusion criteria will be helpful. If a wide range of types of publications were considered, the authors could have used the JBI Critical
Appraisal Checklists because the JBI Scientific Committee had designed specific checklists for all types of studies. ${ }^{7,8}$

Systematic reviews are different and more valid than other literature reviews because they provide the best evidence available to researchers. This systematic review should provide an explicit and repeatable methodology. Therefore, it is recommended that physicians, researchers, and journals follow the PRISMA guidelines because they improve the quality of reports of such studies.

\section{Conflict of Interest Statement}

The authors have no conflicts of interest to declare.

\begin{abstract}
About the Authors
Tabriz Health Services Management Research Center, Health Management and Safety Promotion Research Institute, Tabriz University of Medical Sciences, Tabriz, Iran (Amir-Behghadami, Janati); Iranian Center of Excellence in Health Management (IceHM), Department of Health Service Management, School of Management and Medical Informatics, Tabriz University of Medical Sciences, Tabriz, Iran (Amir-Behghadami, Janati); and Student Research Committee (SRC), Tabriz University of Medical Sciences, Tabriz, Iran (Amir-Behghadami).
\end{abstract}

Correspondence and reprint requests to $\mathrm{Mr}$ Mehrdad Amir-Behghadami, Iranian Center of Excellence in Health Management, School of Management and Medical Informatics, Tabriz University of Medical Sciences, University Rd, Golbad, EAZN 5165665811, East Azerbaijan, Tabriz, Iran (e-mail: behghadami.m@gmail.com).

\section{REFERENCES}

1. Ingrassia PL, Pigozzi L, Bono M, et al. Use of simulated patients in disaster medicine training: a systematic review. Disaster Med Public Health Prep. 2020;epub:1-6.

2. Aromataris E, Riitano D. Systematic reviews: constructing a search strategy and searching for evidence. Am J Nurs. 2014;114(5):49-56. 
3. Gholizadeh M, Amir-Behghadami M, Janati A. Systematic reviews: are they actually well conducted and reported in accordance with PRISMA? Bull Emerg Trauma. 2020;8(1):51-52.

4. Moher D, Liberati A, Tetzlaff J, Altman DG. Preferred reporting items for systematic reviews and meta-analyses: the PRISMA statement. Ann Intern Med. 2009;151(4):264-269.

5. Amir Behghadami M, Janati A. How to report systematic review studies: a critical review of some methodological issues and recommendations to consider when conducting these studies. Bull Emerg Trauma. 2019;7(4):429-430.
6. Janati A, Amir-Behghadami M, Arab-Zozani M. Letter to the editor on the article "Cement augmentation of sacroiliac screws in fragility fractures of the pelvic ring - a synopsis and systematic review of the current literature." Injury. 2020;51(4):1138-1139.

7. Behghadami MA, Janati A. A critical appraisal of the review study to improve its reporting quality. Iran J Nurs Midwifery Res. 2019;24(4): 313

8. Porritt K, Gomersall J, Lockwood C. JBI's systematic reviews: study selection and critical appraisal. Am J Nurs. 2014;114(6):47-52. 